as elution gas hydrogen, nitrogen or carbon dioxide. By taking a convenient standard, an accuracy of about 4 per cent can be obtained.

Details of the apparatus, of the experimental data and the calculations will be published elsewhere.

One of us (A. L.) is greatly indebted to Dr. A. J. P. Martin and to his staff of the Medical Research Council for information and advice given to him in the building of the gas-density balance meter.
A. LIBERTI
L. ConTr
V. CRescenzi

Istituto di Chimica Generale e Inorganica, Università di Roma.

'Martin, A. J. P., and James, A. T., Biochem. J., 63, 138 (1956).

\section{An Unexpected Catalytic Dehydrogenation}

DURING the course of an investigation which is being made on the decomposition of amines in the presence of hydrogen over evaporated metal-film catalysts, an unexpected example of selective catalytic action has been observed. The decomposition of cyclohexylamine on evaporated platinum films at $134^{\circ} \mathrm{C}$. was expected to produce cyclohexane and ammonia according to the equation

$$
\mathrm{C}_{6} \mathrm{H}_{11} \mathrm{NH}_{2}+\mathrm{H}_{2} \rightarrow \mathrm{C}_{6} \mathrm{H}_{12}+\mathrm{NH}_{3}
$$

The reaction was followed by continuous mass. spectrometric analysis of the reacting gases by a technique described elsewhere ${ }^{1}$. As the ions of mass $99\left(\mathrm{C}_{6} \mathrm{H}_{11} \mathrm{NH}_{2}{ }^{+}\right)$decreased in number, ions of mass 17 $\left(\mathrm{NH}_{3}{ }^{+}\right)$appeared but very few ions of mass 84 $\left(\mathrm{C}_{6} \mathrm{H}_{12}{ }^{+}\right)$were observed. A search throughout the mass-range covered by the spectrometer indicated that large quantities of ions of mass 78 were being produced, suggesting that the primary product of the reaction was benzene $\left(\mathrm{C}_{6} \mathrm{H}_{6}\right)$ rather than cyclohexane. Now the position of the equilibrium ${ }^{2}$ in the reaction

$$
\mathrm{C}_{6} \mathrm{H}_{12} \rightleftharpoons \mathrm{C}_{6} \mathrm{H}_{6}+3 \mathrm{H}_{2}
$$

is well over on the side of cyclohexane under the conditions used in this work. The initial reaction mixture consisted of $1.8 \mathrm{~mm}$. cyclohexylamine and $23 \mathrm{~mm}$. hydrogen and, assuming complete conversion according to reaction (1) and the known equilibrium constant for reaction (2), the maximum pressure of benzene to be expected at $134^{\circ} \mathrm{C}$. was only about $10^{-3} \mathrm{~mm}$. The identification of the source of the mass 78 ions as benzene was therefore considered tentative until it was found that when most of the cyclohexylamine was used up the ions of mass 78 began to disappear again and in turn substantial quantities of ions of mass 84 were formed. This showed that the normal catalytic hydrogenation of benzene to cyclohexane, to be expected on a metal like platinum, was then taking place. The formation of benzene was also confirmed by stopping a typical reaction and identifying the benzene in the reaction mixture by vapour-phase chromatography. The whole course of the reaction is shown in Fig. 1.

The initial reaction can be written as

$$
\mathrm{C}_{8} \mathrm{H}_{11} \mathrm{NH}_{2} \rightarrow \mathrm{C}_{6} \mathrm{H}_{6}+\mathrm{NH}_{3}+2 \mathrm{H}_{2}
$$

which is the sum of reactions (1) and (2). Although the equilibrium constant for reaction (1) is not known, it is probably of similar magnitude to that

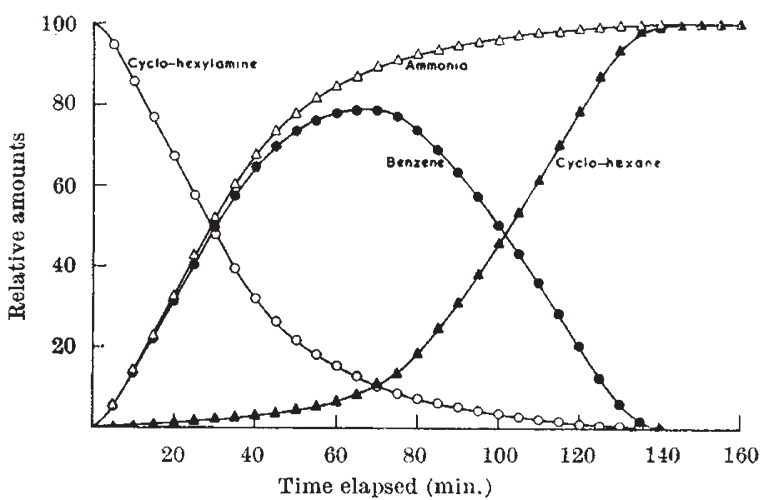

Fig. 1. Relative amounts of cyclohexylamine, ammonia, benzene and cyclohexane during reaction of $1.8 \mathrm{~mm}$. cyclohexylamine and $23 \mathrm{~mm}$. hydrogen on a $5.65 \mathrm{mgm}$. evaporated platinum catalyst at $134^{\circ} \mathrm{C}$.

for the corresponding hydrogenation of methylamine to methane and ammonia which is approximately $10^{12}$ at $134^{\circ} \mathrm{C}$. Combining this figure with the known equilibrium constant ${ }^{2}$ for reaction (2), a value of $10^{13}(\mathrm{~mm} \text {. })^{3}$ is found for reaction (3). The magnitude of this value indicates that it is thermodynamically feasible for benzene to be formed according to reaction (3) even in the presence of a large excess of hydrogen.

The selective action of the catalyst causes reaction (3) to take place in preference to reaction (1). It is not perhaps surprising that benzene should be produced on the surface of the catalyst since it is known that saturated hydrocarbons can lose hydrogen atoms on transition metal catalysts in this temperature-range. However, it is most interesting that the presence of the amine can inhibit the hydrogenation of benzene which is usually a facile catalytic reaction. Further work is in progress to elucidate the details of this type of phenomenon in connexion with the vapour-phase decomposition of cyclic amines on evaporated metal-film catalysts and will be published in due course.

One of us (R. L. M.) is the holder of a British Celanese studentship.

R. L. Moss

C. KembaLL

Department of Chemistry,

The Queen's University of Belfast. July 19.

${ }^{1}$ Kemball, C., Proc. Roy. Soc., A, 207, 539 (1951).

${ }^{2}$ Janz, G. J., J. Chem. Phys., 22, 751 (1954).

\section{Benzene-soluble Aryldiazonium Salts}

WhILE typical salts are soluble in water and insoluble in hydrocarbons a few are soluble in both media. No member of the large and important group of diazonium salts is known to be soluble in weakly polar media, however, and this has prevented a study of the reaction of the salts with such reagents as the organometallic compounds, though their complexes with metallic salts have been used ${ }^{\mathbf{1 , 2}}$.

We have now found that $p$ - $n$-decyloxybenzenediazonium toluene- $p$-sulphonate, $p$-cetyloxybenzenediazonium chloride and $2: 5$-di-n-p-decyloxybenzenediazonium chloride are all moderately soluble in warm benzene, though sparingly in the cold. The salts appear to exist in benzene as ion pairs, though 